\title{
Motivational Interviewing enhances the adoption of water disinfection practices in Zambia
}

\author{
Thevos A, Quick $R$ and Yanduli V
}

These studies represent the first adaptation of the Motivational Interviewing (MI) behavior change approach in the developing world, using health workers directly from the community. The objective was to compare the effectiveness of the standard practice of health education (comparison group) to MI (experimental group) in initiating and sustaining safe water treatment and storage behavior. Methods: focus groups and community surveys were conducted prior to health worker training. The main outcome variables were detectable disinfectant levels in stored household water (for Field Trial 1) and disinfectant sales (for Field Trial 2). Results: in Field Trial 1 (n $=185$ households), a very high adherence rate was achieved (range 71.1-94.7\%), with no statistical differences between the groups. Field Trial 2 ( $n=427$ households) incorporated lessons learned from the previous trial and resulted in much higher purchase rates of the disinfectant in the MI group, $\mathrm{t}(7)=10.69, \mathrm{p}<0.001$, eta ${ }^{2}=$ 0.94. Conclusion: MI intervention appears promising for public health initiatives in the developing world. Further work in this area is indicated.

Key Words: behavior therapy, developing countries, Motivational Interviewing, water purification.

\section{INTRODUCTION}

In the developing world, diarrheal disease is a leading cause of morbidity and mortality among children less than 5 years old. Water is an important vehicle for the transmission of enteric pathogens; it has been estimated that over one billion people lack access to safe water (Centers for Disease Control, 1990; Bern et al., 1992). In response to this urgent problem, the Centers for Disease Control and Prevention (CDC) and the Pan American Health Organization developed a simple, inexpensive, easily disseminated, and effective intervention for safe water treatment and storage at the household level (Mintz and Tauxe, 1995).

The intervention involves household disinfection with a locally produced sodium hypochlorite solution, and education on proper storage of treated water and disinfectant solution, the causes of diarrhea, and the importance of diarrhea prevention. A series of studies in Bolivia demonstrated that households using this water intervention have significantly better water quality and a lower incidence of diarrhea than control households (Quick et al., 1996, 1999). Given the success of the water intervention in Latin America, two field evaluations extending to the African continent were conducted in two peri-urban communities of Kitwe, Zambia between March 1998 and January 1999. For these trials, the education element was augmented by a novel behavior change component, Motivational Interviewing, to investigate its effectiveness in improving adherence rates in the use of this safe water treatment and storage system.

\section{BACKGROUND}


Work in the behavioral sciences has produced efficacious, brief intervention techniques to produce rapid, internally motivated behavior change. One such approach, developed by Miller and Rollnick, is termed Motivational Interviewing (Miller and Rollnick, 1991). Motivational Interviewing (MI) is grounded in decisionmaking theory and motivational psychology. It incorporates the trans-theoretical model of the stages of change, which conceptualizes change as a process. People move through stages with corresponding different levels of readiness (Prochaska and DiClemente, 1984, 1992; Prochaska and Velicer, 1997). The first stage, 'precontemplation', applies when the person is not ready to consider change or is unaware of any need to change. 'Contemplation', the next stage, implies ambivalence and relates to the person who both considers change and rejects it. The 'preparation' stage refers to people open to changing and those who may be preparing to make a change. An individual who is actually engaging in actions with the intention of bringing about change is said to be in the 'action' stage.

Reports of stage-based interventions have shown that employing particular strategies for different stages can be quite effective in the successful changing of health behaviors (DiClemente et al., 1991; Perz et al., 1996; Prochaska and Velicer, 1997). Conversely, employing strategies, e.g. advice giving or suggestions for taking action with a person who is not ready for change can be premature, inappropriate and ineffective. A more person-centered, stage-based approach, e.g. MI, with the goal of resolving ambivalence and eliciting a person's own arguments for change has been shown to be more effective, particularly among those less ready to change (Heather et al., 1996). The MI practitioner employs motivational strategies to mobilize a person to decide to choose a change in behavior, and to use his or her own resources to do so. Its defining feature is a style of communicating whereby the practitioner takes a partnership role with the client (Rollnick and Miller, 1995).

Motivational Interviewing has been effective in helping clients change behaviors in several health-related areas, including alcohol and other drug abuse (Bien et al., 1993; Miller et al., 1993; Saunders et al., 1995; Heather et al., 1996; Project MATCH Research Group, 1997; Project MATCH Research Group, 1998; Daley et al., 1998), cardiovascular health (Woollard et a/., 1995), and diabetes (Smith et al., 1997; Trigwell et ai, 1997). Because MI is both client-centered and effective in producing healthy changes in a wide range of behaviors, it was a promising approach for use in the present studies.

The two field trials reported here represent the first application of MI principles in the developing world using health workers directly from the community. The objective was to compare the effectiveness of the standard practice of health education (Ed Only) to Motivational Interviewing (MI) in initiating and sustaining safe water treatment behavior.

\section{METHODS}

The communities of Ipusukilo and Luangwa, both low socioeconomic status (SES), peri-urban communities of Kitwe, Zambia were selected as the study sites for this project because they lacked a water system and, in a survey conducted by BASICS (a United States Agency for International Development-funded project), had identified drinking water safety as a major concern. The communities are served by volunteers in their respective Neighborhood Health Committees (NHC). NHC 
members (NHCs) provide health information to their neighbors and have regular meetings at the local Health Clinics.

\section{Baseline surveys}

Before the behavior change intervention trials were implemented, Zambian nurses from the Health Clinics in the two communities conducted a baseline survey of all residents in the sample, asking about demographic characteristics, and water handling and sanitary habits. Separate focus groups and individual interviews with community residents were also conducted by the study team to generate information regarding the health beliefs of the study community. Stored water from all study households and source water from a random sample of $25 \%$ of households were tested for free and total chlorine residuals, using the $\mathrm{N}, \mathrm{N}$-diethyl-phenylenediamine (DPD) method, and for contamination with Escherichia coli, using membrane filtration (Mates and Shaffer, 1989).

\section{Informed consent}

Informed consent in accordance with the requirements of the CDC Institutional Review Board and the Ethics Committee of the Tropical Diseases Research Centre of Zambia (TDRC) was obtained from all study participants.

\section{Field Trial 1: Ipusukilo community}

Field Trial 1 took place from March to June 1998. A microenterprise for the production, bottling, sale and distribution of the sodium hypochlorite (chlorine) disinfectant was established at the Ipusukilo Health Clinic using a SANILEC on-site hypochlorite generator (Exceltech International, Sugarland, TX, USA). Three NHCs were trained in the operation and maintenance of the SANILEC.

Households ( $n=188$ ) were randomly selected from two zones in Ipusukilo. In the 2 weeks between the baseline survey and the beginning of the Ipusukilo intervention, three families moved out of the community, leaving 185 households at the start of the intervention. The behavior change intervention study was designed with two experimental groups: an intervention group (MI, $n=92$ ) and a comparison group (Ed Only, $n=93$ ). The nurses from the Ipusukilo Health Clinic selected the 10 most active NHCs and assigned them to two groups of five to deliver the intervention and comparison conditions. Households were assigned to NHCs based on proximity to each NHC's residence. This assignment strategy resulted in households within the same neighborhood being assigned to different experimental conditions. In compensation for their participation in the study, each household received disinfectant free for the duration of Field Trial 1.

\section{Training of NHCs}

At the beginning of the study, all 10 of the NHCs participated in a workshop about the causes of diarrhea, the importance of diarrhea prevention, and the proper use of chlorine disinfectant and storage of treated water. This training was provided to the $\mathrm{NHCs}$ by the study team so that the NHCs could, in turn, educate the members of the study households and reinforce this information as needed at subsequent household visits. NHCs were given brochures and educational flip charts which presented this information in simple illustrations and text in English (the official national language) and Bemba (the local language). In addition, all of the NHCs received instruction in basic interviewing skills, the design and rationale of the study, and a review of the general procedures in the completion and submission of study rating forms which were required to document household visits. The workshop and 
informational materials were the only tools that the five NHCs in the Education Only comparison group received.

\section{Motivational Interviewing training}

The MI intervention group consisted of three local nurses and five NHCs who were trained in the theoretical model of the stages of change, the basic principles employed in brief Motivational Interviewing to effect behavior change, and the task components relevant to these. The principles of MI involve expressing empathy, developing discrepancy, avoiding argumentation, rolling with resistance, and supporting self-efficacy. The MI tools are open-ended questions, affirmations, reflective listening and summarizing (Miller and Rollnick, 1991; Miller et al., 1992). The nurses, all of whom were English speaking, were trained first and separately from the NHCs by the behavioral scientist on the study team. The nurses then developed and delivered the MI training to the NHCs. A total of $10 \mathrm{~h}$ over 4 days were devoted to the nurses' training.

The MI content was adapted to be relevant to water and sanitation issues as well as cultural considerations based on information gained from focus groups, individual interviews and guidance from the local nurses. The training utilized a participatory workshop format with didactic instruction and exercises employing role-plays and MI tools.

Printed material relevant to the MI training was translated into Bemba for distribution to the NHCs. The series of workshops was designed to be temporally close enough to allow for skill and information retention. Although 10-12 h of NHC training were planned, only $5 \mathrm{~h}$ were actually accomplished in three separate sessions over a 2 -week period due to illness and competing commitments. The first two trainings (each was $2 \mathrm{~h}$ long) occurred 2 days apart; the third took place 2 weeks after the second.

\section{Outcome measure}

The main outcome variable was whether a household had a detectable level of total chlorine in its stored water. Zambian health workers from TDRC tested stored water in all study households for free and total chlorine residuals. Water testing occurred on four occasions, $\sim 2$ weeks apart. Because free chlorine levels decline over time in stored water, and we could not guarantee that our visits would occur at a defined period after the household filled their water vessel, we decided that detectable total chlorine residual was the best indicator of adherence, i.e. evidence that the household was dosing their water with the chlorine disinfectant.

\section{Follow-up survey}

At week 9, a brief follow-up interview about water-handling practices, and knowledge of causes and prevention of diarrhea was conducted with study participants.

\section{Field Trial 2: Luangwa community}

A second study was implemented in the community of Luangwa in June 1998 using lessons learned from the previous trial in Ipusukilo. Two clearly defined zones were selected by community nurses as treatment zones. Households in one zone received only health education (Ed Only, $n=200$ ) and households in the other zone received MI $(n=132)$. With this assignment procedure, neighbors were not assigned to two different study conditions and the risk of information sharing was consequently reduced. In this field trial, the MI training was delivered for $4 \mathrm{~h}$ each on two 
consecutive days to five NHC members and to a local nurse. The behavioral scientist had no contact with the comparison group of five NHCs who delivered the Ed Only treatment. This was done in order to avoid inadvertent teaching of interviewing skills to this group, thereby diluting the difference between the treatment conditions.

A microenterprise for the sale of sodium hypochlorite solution was established in the Luangwa Health Clinic. Sodium hypochlorite solution sales to the Ed Only and MI zones were recorded by the nurses at the Luangwa Health Center. The number of bottles sold divided by the number of possible buyers (i.e. households) in the community was calculated for each month ( 8 months total). A paired samples $t$-test was conducted to compare sales ratios for the two treatment groups. Because of funding limitations, household level testing of free and total chlorine residuals could not be performed.

Some of the households in the MI zone ( $n=95,72 \%)$ participated in weekly diarrheal surveillance during Field Trial 1 (Group A). Of concern was whether these households were sensitized to buy more chlorine because of their inclusion in the diarrheal surveillance sample, and thus inflated the MI group effect. This concern led us to incorporate a group that did not participate in the diarrhea surveillance. A paired samples $t$-test was therefore conducted to compare sales ratios between the surveillance group (Group A) and the remaining MI households (Group B) where monthly sales data were available for each ( 6 months, August-January).

\section{RESULTS}

\section{Field Trial 1: Ipusukilo community}

In Field Trial 1, sodium hypochlorite utilization rates and water-handling practices were compared between the MI and Ed Only groups using the $t$-test. Data from 166 households were available at the end of this 8 -week study. During the course of the study 19 households were excluded: 18 moved outside the study area and one refused to participate further. Results of the baseline survey indicated that the Ed Only and MI groups were not significantly different (alpha $=0.05$ ) on demographic variables and baseline safe water practices. The mean number of persons/household was 5.8 (SD = 2.7), the median age of respondents was 33 (range 14-89) and the median annual per capita income was $\$ 608$ (range \$49-3650). Seventy-seven percent of the respondents were female. All respondents reported using a shallow well as the source for drinking water. Forty percent of the entire sample at baseline reported that they had previously treated their household water to make it safe. Only $9.8 \%$ of households had treated their water in the past day and $7 \%$ within the past week. All households used a latrine. Fewer than $4 \%$ of households reported having electricity.

The testing for detectable total chlorine residuals revealed a high rate of overall adherence, ranging from 71.1 to $94.7 \%$ of households having positive chlorine readings over the 8 weeks of sampling (Figure 1 ). Adherence rates for the MI and Ed Only groups were not significantly different at any time point.

Fig. 1: Percent of households with detectable total chlorine residuals in stored water, by intervention (Motivational Interviewing) and comparison (Education Only) groups, 
Field Trial 1, Ipusukilo community, Kitwe, Zambia, April-May 1998.

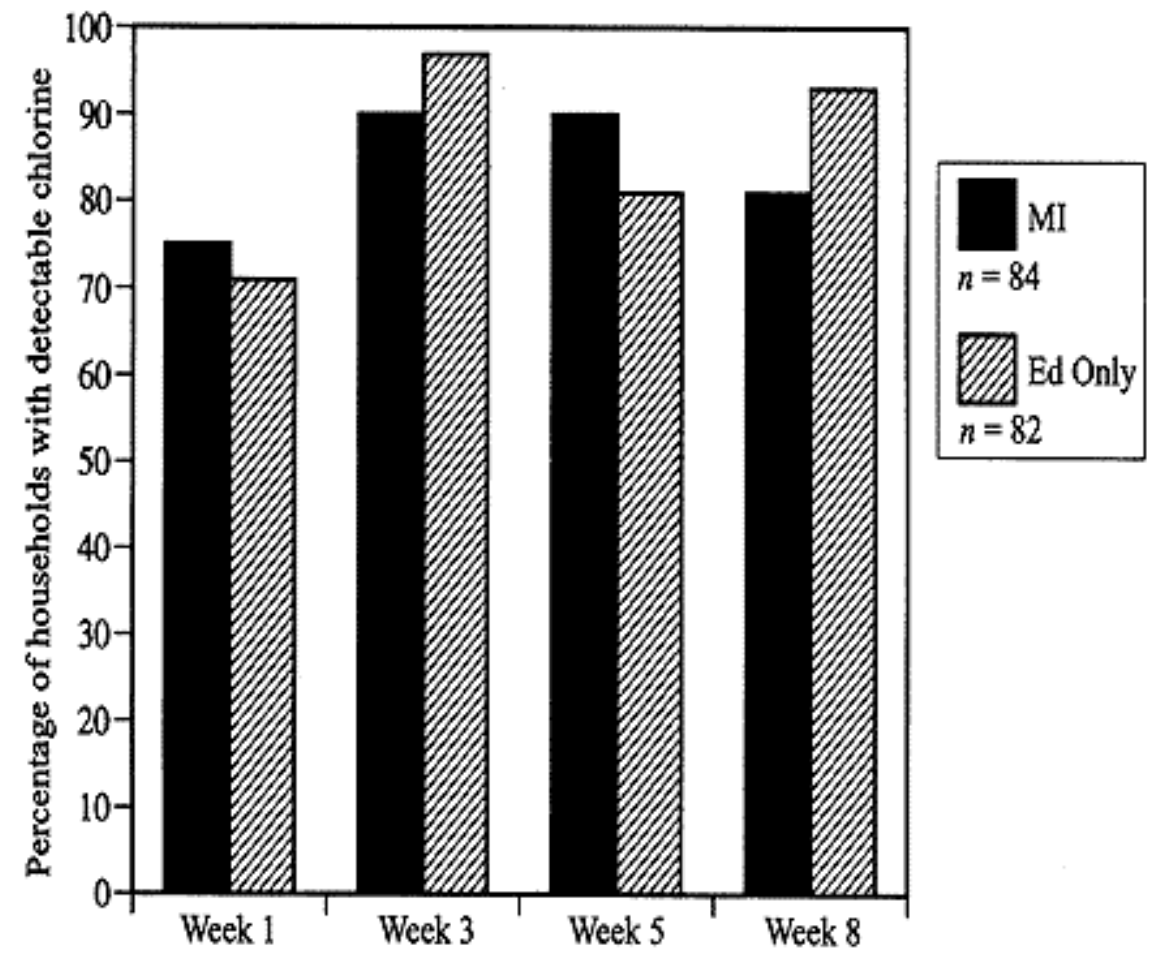

During the follow-up survey, $96.9 \%$ of respondents reported that they used chlorine disinfectant to treat their water. Similarly, direct observation indicated excellent adherence with instructions for handling the disinfectant, as 99\% kept chlorine out of the sun, $96 \%$ kept chlorine out of the reach of children, $98 \%$ knew the correct dose of chlorine for their container size, and $90 \%$ stated an intention to continue to use chlorine. Those who did not intend to purchase chlorine cited lack of money as the reason. None of these safe water-handling indicators revealed statistically significant differences between the MI and Ed Only groups.

\section{Field Trial 2: Luangwa community}

The demographics of the Luangwa community closely parallel those of Ipusukilo, and there were no differences between the two treatment groups. The mean number of persons/household was $6.2(S D=3.0)$, the median age of respondents was 28 (range 10-79) and the median annual per capita income was $\$ 406$ (range \$943650 ). Seventy-two percent of the respondents were female. Fewer than $5 \%$ of households reported having electricity.

Overall, the MI zone had significantly higher sales ratios than the Ed Only zone, $t(7)$ $=10.69, p<0.001$ (Figure 2). The effect size is large, eta ${ }^{2}=0.94$, indicating that $94 \%$ of the variance in sales ratios is attributable to treatment group assignment. Within the MI zone, Group A ( $n=95$ households) and Group B ( $n=37$ households), did differ significantly, $t(5)=6.49, p=0.001$, although not in the direction expected. That is, the households who participated in the diarrheal surveillance had lower sales ratios than the comparison group. While this finding was unexpected, it provides an even stronger assurance that the mixing of Group A and Group B households in the MI zone did not confound the data demonstrating a strong association between use of MI and disinfectant sales. 
Fig. 2: Magnitude of sales of bottles of sodium hypochlorite solution (no. of bottles sold/no. of households), by intervention (Motivational Interviewing) and comparison (Education Only) zones by month. Field Trial 2, Luangwa community, Kitwe, Zambia, June 1998-January 1999.

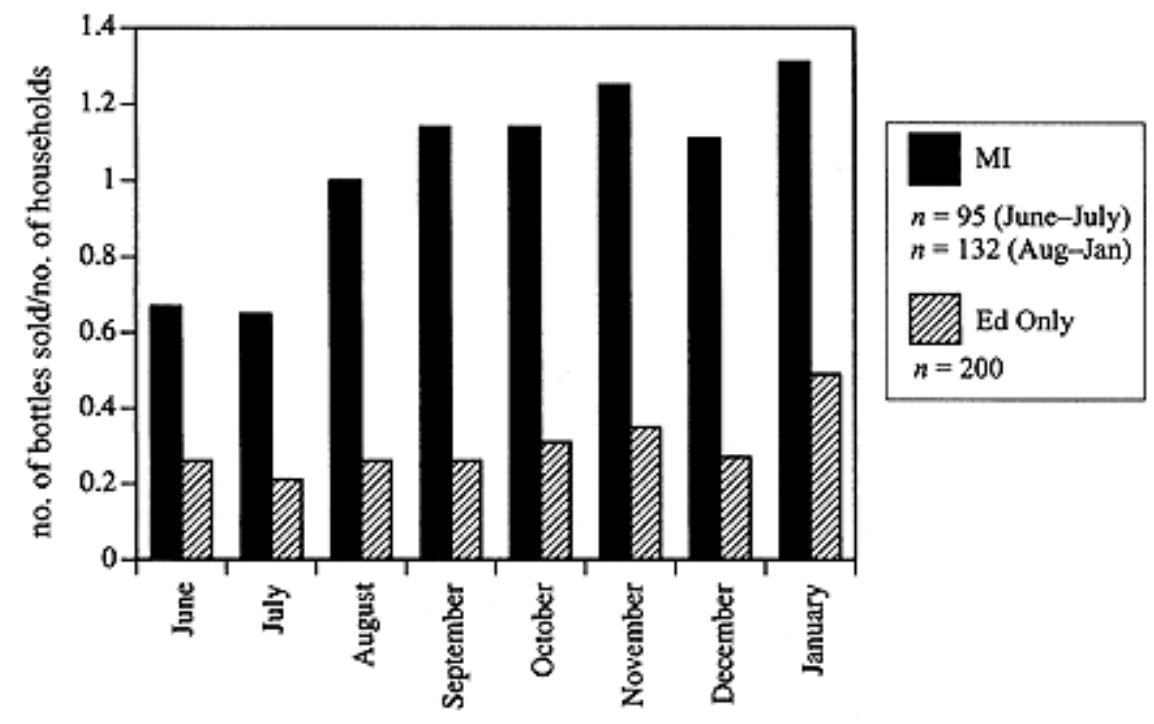

\section{DISCUSSION}

These field trials were the first to employ the novel behavior change intervention technique of Motivational Interviewing to prevent diarrheal diseases in a developing country. In Field Trial 1, despite methodological problems, there was a very high rate of adherence and community acceptance of the water intervention as evidenced by the high percentage of water samples with detectable chlorine. In previous Bolivian field trials of this water quality intervention in which only an education component was used, adherence rates were significantly lower (Quick et al., 1999).

The rates of adherence in Field Trial 1 were equally high in both the MI intervention and the Ed Only comparison groups, with no statistically significant difference between the groups. There are several possible explanations for these findings. First, at baseline, many participants indicated that they had some education about water contamination, causes of diarrhea and methods of its prevention. Second, the study community placed a high priority on water quality, which, combined with education about water contamination and diarrhea, could contribute to high levels of readiness or motivation to adopt the water intervention. Put another way, it may be that people were more in the 'action' stage of change. MI is a strategy which is aimed at resolving ambivalence towards behavior change. However, in this population, little ambivalence was present. Further, people in the 'preparation' or 'action' stages of change often respond to skill building. The educational brochures describing the proper use of the water intervention may have been all that was needed to render these skills, and this was provided equally in both of the behavior change conditions. Third, chlorine disinfectant was available free of charge during the study. Because no monetary cost was involved and there was little effort needed for the participants to receive the product, the ideal circumstances to measure motivation for long-term, sustained behavior change may not have been present. Fourth, the lack of group differences may have been related to training efforts. All of the NHCs received instruction in elementary interpersonal interviewing skills, e.g. exclusively attending 
to the respondent, not interrupting, building rapport and listening carefully. These basic interviewing practices may have influenced the adherence rates and resulted in a ceiling effect which made group differences undetectable. To avoid this potentially confounding factor, in Field Trial 2 only the MI group received interpersonal interviewing instruction. Lastly, problems were encountered in the MI training during Field Trial 1 . Scheduling difficulties and lack of attendance due to family illnesses greatly hampered efficient progress. On five separate occasions, NHCs' training sessions had to be canceled and rescheduled. When trainings did occur, an extensive amount of time was needed to review because so much time had elapsed since the previous meeting. This made actual practice exercises, which are critical, difficult to realize. Thus, although the NHCs and nurses were instructed in the key concepts and principles of MI, the amount and continuity of trainings, degree of practice within trainings, as well as time left for supervision and retraining in the field, were less than ideal. For these reasons it may be that in Field Trial 1, the comparison and intervention conditions were more similar than different, and therefore there was neither a true comparison group nor an adequate intervention group which could be compared.

In Field Trial 2, some of these issues were addressed. Training in MI was improved. Specifically, the extended length of the study allowed for valuable in-field instruction and guided practice opportunities specifically tailored to individual needs. Also, contact by the behavioral scientist with the comparison condition NHCs was eliminated. The intervention was aimed at encouraging households to purchase chlorine, rather than simply to utilize free chlorine. Chlorine sales may be a measure that is more sensitive to and representative of intrinsic behavior change as it is a more realistic measure of motivation and adherence with the water intervention, particularly in a low SES group. Unfortunately, because of a lack of adequate funding, we were unable to test residual chlorine levels, which is a more objective and precise measure of adherence.

These findings may provide support for using theory-driven behavioral change interventions in the developing world (Stanton et al., 1992; Stanton, 1997). Results from Field Trial 2 are remarkably promising for the potential of MI to effect long-term behavior change in at-risk populations. Rates of purchase of sodium hypochlorite solution were much higher in the MI group than the Ed Only group and were sustained during the 8-month course of the trial. This effect is remarkable considering that the residents had to change their daily behavior as well as expend extremely limited resources to purchase the disinfectant. Most importantly, an efficacy study conducted concurrently with Field Trial 1 showed that the sodium hypochlorite point-of-use disinfection method reduced diarrhea incidence (Quick, Centers for Disease Control and Prevention, unpublished data), which is consistent with previous work accomplished in Latin America (Quick etai, 1999).

Based on results from these trials, Motivational Interviewing deserves more thorough scientific investigation to determine its efficacy in preventing disease in the developing world. There is also a need to refine implementation methods. For example, weekly individualized visits may not be practical for most behavioral change interventions which are implemented on a community level. The Zambian infrastructure provided by the NHCs network is exceptional and very useful in this regard. More research is needed to attempt to identify the salient features of MI and/or other brief interventions to allow for less frequent, briefer contacts and those 
that can be applied to whole communities, with special emphasis on the major health problems faced by developing countries.

\section{ACKNOWLEDGEMENTS}

Funding for this project was provided by the Office of Health Communication, National Center for Infectious Diseases, Centers for Disease Control and Prevention and the United States Agency for International Development. The authors would like to acknowledge Dr Akiko Kimura, the Tropical Diseases Research Centre of Zambia, the Kitwe City Council, Mr Peter Kalenga, Mr Promise Kaminsa, Dr Cleto Chashi, and the Urban Health Program of BASICS for technical support, and the nurses who participated in the trial: Margaret Bowa, Catherine Gondwe, Dalley Kafwimbi, Rachel Lungu, Irene Musungu and Joyce Ndhlovu. The Neighborhood Health Committee workers, who were central to this endeavor, are also gratefully acknowledged.

\section{REFERENCES}

1. Bern, C, Marlines, J., de Zoysa, I. and Glass, R. (1992) The magnitude of the global problem of acute diarrheal disease: a ten-year update. Bulletin of the World Health Organization: 70: 705-714.

2. Bien, T. H, Miller, W. R. and Tonigan, J. S. (1993) Brief interventions for alcohol problems: a review. Addiction: 88: 315-335.

3.Centers for Disease Control (1990) Waterborne disease outbreaks, 1986-1988. Morbidity and Mortality Weekly Report: 39: 1-13.

4.Daley, D. C., Salloum, I. M., Zuckoff, A., Kirisci, L. and Thase, M. E. (1998) Increasing treatment adherence among outpatients with depression and cocaine dependence: results of a pilot study. American Journal of Psychiatry: 155: 16111613.

5. DiClemente, C. C, Prochaska, J. 0., Fairhurst, S. K., Velicer, W. R, Velasquez, M. M. and Rossi, J. S. (1991) The processes of smoking cessation: an analysis of precontem-plation, contemplation, and preparation stages of change. Journal of Consulting and Clinical Psychology: 59: 295-304.

6. Heather, N., Rollnick, S., Bell, A. and Richmond, R. (1996) Effects of brief counselling among male heavy drinkers identified on general hospital wards. Drug and Alcohol Review: 15: 29-38.

7. Mates, A. and Shaffer, M. (1989) Membrane filtration differentiation of $E$. coli from coliforms in the examination of water. Journal of Applied Bacteriology: 67: 343-346.

8. Miller, W. R., Benefield, R. G. and Tonigan, J. S. (1993) Enhancing motivation for change in problem drinking: a controlled comparison of two therapist styles. Journal of Consulting and Clinical Psychology: 61: 455-461.

9. Miller, W. R. and Rollnick, S. (1991) Motivational Interviewing: Preparing People to Change Addictive Behavior. The Guilford Press, New York. 
10. Miller, W. R., Zweben, A., DiClemente, C. C. and Rychtarik, R. G. (1992) Motivational enhancement therapy (MET): a clinical research guide for therapists treating individuals with alcohol abuse and dependence (DHHS Publication No. ADM 92-1894). US Government Printing Office, Washington, DC.

11. Mintz, E. D. and Tauxe, R. V. (1995) Safe water treatment and storage in the home: a practical new strategy to prevent waterborne disease. Journal of the American Medical Association: 273: 948-953.

12. Perz, C. A., Di Clemente, C. C. and Carbonari, J. P. (1996) Doing the right thing at the right time? The interaction of stages and processes of change in successful smoking cessation. Health Psychology: 15: 462-468.

13. Prochaska, J. 0. and Di Clemente, C. C. (1992) Stages of change in the modification of problem behaviors. Progress in Behavior Modification: 28: 183-218.

14. Prochaska, J. 0. and DiClemente, C. C. (1984) Toward a comprehensive model of change. In Miller, W. R. and Heather, N. (eds) Treating Addictive Behaviors:

Processes of Change. Plenum Press, New York, pp. 3-27.

15. Prochaska, J. 0. and Velicer, W. F. (1997) The trans-theoretical model of health behavior change. American Journal of Health Promotion: 12: 38-48.

16. Project MATCH Research Group (1997) Matching alcoholism treatments to client heterogeneity: Project MATCH posttreatment drinking outcomes. Journal of Studies on Alcohol: 8: 7-29.

17. Project MATCH Research Group (1998) Matching alcoholism treatments to client heterogeneity: Project MATCH three-year drinking outcomes. Alcoholism: Clinical and Experimental Research: 22: 1300-1311.

18. Quick, R. E., Venczel, L. V, Gonzalez, O., Mintz, E. D., Highsmith, A. K., Espada, A., Damiani, E., Bean, N. H., De Hannover, E. H. and Tauxe, R. V. (1996) Narrowmouthed water storage vessels and in situ chlorination in a Bolivian community: a simple method to improve drinking water quality. American Journal of Tropical Medicine \& Hygiene: 54: 511-516.

19. Quick, R. E., Venczel, L. V, Mintz, E. D., Soleto, L., Aparicio, J. and Gironaz, M. (1999) Diarrhea prevention through point-of-use water treatment and storage: a promising new strategy. Epidemiology and Infection: 122: 83-90.

20. Rollnick, S. and Miller, W. R. (1995) What is motivational interviewing? Behavioural and Cognitive Psychotherapy: 23: 325-334.

21. Saunders, W, Wilkinson, C. and Phillips, M. (1995) The impact of a brief motivational intervention with opiate users attending a methadone program. Addiction: 90: 415-424.

22. Smith, D. E., Heckemeyer, C. M., Kratt, P. P. and Mason, D. A. (1997)

Motivational interviewing to improve adherence to a behavioral weight-control 
program for older obese women with NIDDM. A pilot study. Diabetes Care: 20: 5254.

23. Stanton, B. (1997) Editorial: good news for everyone? American Journal of Public Health: 87: 1917-1919.

24. Stanton, B., Black, R., Engle, P. and Pelto, G. (1992) Theory-driven behavioral intervention research for the control of diarrheal diseases. Social Science and Medicine: 35: 1405-1420.

25. Trigwell, P., Grant, P. J. and House, A. (1997) Motivation and glycemic control in diabetes mellitus. Journal of Psychosomatic Research: 43: 307-315.

26. Woollard, J., Beilin, L., Lord, T, Puddey, I., MacAdam, D. and Rouse, I. (1995) A controlled trial of nurse counselling on lifestyle change for hypertensives treated in general practice: preliminary results. Clinical and Experimental Pharmacology and Physiology: 22: 466-468.

\section{Suggested citation:}

Thevos A, Quick R and Yanduli V. Motivational Interviewing enhances the adoption of water disinfection practices in Zambia. Health Promotion International. 2000; 15(3):207214. 